DOI: $\mathrm{xxx} / \mathrm{xxxx}$

\title{
COMMENT
}

\section{Initial Energy of a Spatially Flat Universe - a Hint of its Possible Origin}

\author{
Fulvio Melia*
}

${ }^{1}$ Departments of Physics and Astronomy, and the Applied Math Program, The University of Arizona, Tucson, Arizona, U.S.A.

Correspondence

*Email: fmelia@arizona.edu

The evidence for a Big Bang origin of the Universe is truly compelling, though its cause remains a complete mystery. As the cosmic spacetime is revealed to us with ever improving detail, however, we are beginning to refine the range of its possible initial conditions-at least within the framework of current physical theories. The Universe, it seems, is spatially flat, and here we discuss in clear, straightforward terms why this trait implies a cosmos with zero kinetic plus gravitational energy, though apparently not zero total energy. Such an outcome has far reaching consequences because of the possibility that the Universe may have begun its existence as a quantum fluctuation. Was this from 'nothing,' or perhaps a pre-existing vacuum? A non-zero total energy would seemingly preclude the former scenario, but not necessarily the latter, though this would then raise the question of how a fluctuation with non-zero energy could have lived long enough, or classicalized, for us to see it 13.5 billion years later. The high-precision measurement of the Universe's spatial curvature may thus constitute the first tangible piece of evidence impacting a possible quantum beginning.

\section{KEYWORDS:}

Big Bang; cosmological parameters; cosmology: observations; cosmology: early Universe; cosmology: theory; gravitation

\section{1 | INTRODUCTION}

Over the past half century, many attempts have been made at 'calculating' the energy of the Universe using a variety of approaches with a diverse set of underlying assumptions and definitions. With very few exceptions, these studies have inevitably concluded that the Universe has zero total energy. Given that the definition of what constitutes a viable contribution to this energy has not been uniformly consistent, however, one may question whether all of these treatments can be correct. In fact, with such ambiguity, how can one be certain that any of them are physically meaningful? There is also the suspicion that 'zero energy' is a fundamentally preferred answer because such an initial condition lends considerable support to the notion that the Universe may have started as a quantum event from 'nothing' or, at the very least, from a pre-existing vacuum. These two scenarios are quite different, of course, though the condition of zero energy seems to be a favoured requirement in both cases.

The question of how much energy the Universe possesses first started emerging via a topological argument due to P. Bergmann quoted by Tryon (1973), and then elaborated upon by several other authors, including Albrow (1973), Guth (1981), Rosen (1994), Cooperstock (1994), and several more since then. All along, an important (though not exclusive) motivation for addressing this question has been the proposal first made by Tryon (1973) that our Universe is a fluctuation of the vacuum, to be understood in the context of quantum field theory. Tryon conjectured that the appearance of the Universe 'from nowhere' would require it to have a net zero value for all its conserved quantities, the most important of which is 
energy. He argued that the enormous amount of mass energy in the cosmos is canceled by its (negative) gravitational potential energy, and proceeded to show that these two quantities, contained within the Hubble radius $R_{\mathrm{h}} \equiv c / H$, where $H$ is the Hubble parameter, differ from each other only by a factor of order unity.

Tryon made no mention of the positive energy due to the Universe's expansion, however, and envisaged the initial fluctuation as having taken place in the vacuum of a larger space in which the cosmos is embedded. As such, the total fluctuation energy $\Delta E$ need not have been zero, though one would then have to explain, via the Heisenberg uncertainty principle, $\Delta E \Delta t \sim \hbar / 2$, how the Universe could have lived long enough for us to see it $\Delta t \sim 13.5$ billion years later. Tryon's paper was quite speculative, but it was the seminal work that introduced a quantum origin as a possible explanation for the Big Bang. He even went so far as to point out that a pre-existing vacuum should be unstable against large-scale fluctuations in the presence of a long-range, negative-energy, universal interaction, such as gravity.

To illustrate how broadly this discourse has meandered over the years, it has even been suggested that a pre-existing vacuum would have permitted an enormous range of fluctuations (Šekrst 2020), but intelligent beings would see only a Universe whose outcome is suitable for them to exist. This philosophically interesting argument, known as the anthropic principle (Barrow \& Tipler, 1988), is quite forgiving because only a Universe capable of supporting life would be seen, no matter how statistically improbable it would be compared to other possibilities. But notice that implicit in this argument is the requirement that there be a distribution of possible quantum fluctuations, among which the one that produced our highly improbable Universe was just a representative of many (perhaps an infinite number of) others.

This was also an issue considered in some detail by Ellis (2006), who argued that a choice between various contingent initial conditions somehow occurred at the Big Bang, but it is not clear how that selection came about, at least not in the absence of an anthropic principle. The questions one may ask in this context seem endless, even beyond scientific exploration. For example, why did the Universe's expansion as a bubble from that pre-existing vacuum start when it did, rather than at some previous time in a presumably pre-existent eternity? Attempts at avoiding such difficult or unanswerable queries often avoid a beginning by subscribing to cyclic phases, as in Tolman's series of expansion and collapse cycles (Tolman, 1931), or Linde's eternal chaotic inflation (Linde, 2007) and Khoury et al.'s ekpyrotic universe (Khoury, Ovrut, Steinhardt, \& Turok, 2001).

Several alternative proposals have also been made for initiation events in the absence of space and time, though the distinction between such models, in which literally 'nothing' existed prior to the Big Bang and those with a pre-existing vacuum, is sometimes blurred. The obvious corollary to the former is that both space and time would have been created at the Big Bang along with the Universe itself. This class of cosmological origins includes the 'Universe from Nothing' concept promoted by several workers, such as Krauss (2012), Vilenkin (1982), Zeldovich and Starobinskij (1984) and, more recently, $\mathrm{He}, \mathrm{Gao}$, and Cai (2014). But this approach tends to face greater scrutiny than the pre-existing vacuum idea, for the simple reason that an 'ontic seed' must have been necessary to actually cause the fluctuation, as has been argued by Isham (1994). In addition, this class of models is distinguished from the pre-existing vacuum idea by the absolute requirement of zero energy throughout the Universe's history.

For such reasons, the 'Universe from Nothing' proposals certainly receive more vigorous criticism than their preexisting vacuum counterparts. For example, Kohli (2016) describes several flaws in the fundamental basis of these models, amplifying the point made earlier by Isham. On the one hand, he argues, these models assume that the Universe originated from nothing, yet they require all the complex machinery of variational principles, differential and pseudo-Riemannian geometry, topology, general relativity and quantum field theory, without ever addressing the question of where these come from. Indeed, even the theory used to describe the quantum event itself often requires an underlying superspace, which is absent by default.

Having said this, our goal in this paper is not so much to argue for or against either class of models, but merely to motivate our exploration of what the recent cosmological observations are telling us about the most critical ingredient in all of these scenarios, i.e., the total energy $(E)$ of the Universe. As we saw above, the first attempts (by Bergmann and Tryon) were rather simple, and probably unrealistic. For example, Bergmann's closed universe would not be consistent with the spatial flatness we are measuring today. And, as noted earlier, Tryon did not actually demonstrate that $E$ is exactly zero, partly because he did not include all possible contributions to it. But these were only the first forays; there have been many others since then.

Nevertheless, attempts at calculating the energy of the Universe prior to the 2000's were largely based on the use of pseudotensors, rendering those approaches highly questionable (Faraoni \& Cooperstock, 2003). Pseudotensors may be useful tools in studies of bounded systems that are asymptotically Minkowskian, but the Universe is not bounded for Friedmann-Lemaittre-Robertson-Walker (FLRW) metrics with a spatial curvature constant $k \leq 0$ (see Eq. 1 below). A more reasonable proposal for calculating $E$ with a full general relativistic treatment was introduced by Faraoni and Cooperstock 
(2003), who showed that $E$ is constant for $k \leq 0$. They then argued that these universes are asymptotically Minkowskian and must therefore have a vanishing energy, concluding that $E=0$ from the beginning.

But it is not clear that this argument works for an FLRW spacetime with $k \neq 0$, since it is not asymptotically Minkowskian when expressed in terms of comoving coordinates, though one can demonstrate that it is equivalent to Minkowski space with an appropriate gauge transformation (Melia, 2012). Thus, one may conclude for $k \neq 0$ that $E$ is constant, but not that it is also zero. For example, Kohli (2016) points out that any spatially homogeneous and non-static universe, i.e., one that does not contain a global timelike Killing vector, is necessarily not asymptotically flat. On the other hand, their result for $k=0$ appears to agree with one of our conclusions in this paper, and we shall return to this in $\S$ IV below. It should be pointed out, though, that Faraoni and Cooperstock did not include all possible contributions to $E$. They based their approach on the use of a Lagrangian, from which they derived the Hamiltonian, so they certainly considered both the 'kinetic' and 'potential' energy components, but did not include the cosmic fluid's energy density $\rho$ itself in the overall energy budget.

In this paper, we shall take a different-much more pedagogical-approach to interpret the value of $E$, based on the observational determination that our Universe is spatially flat (i.e., $k=0$ ). We shall focus our attention on the most reliable 'energy conservation' equation we have for the FLRW spacetime, i.e., the Friedmann equation (see Eq. 2 below). We shall interpret its meaning using a Newtonian approach, though strongly motivated by the Birkhoff-Jebsen theorem (Birkhoff \& Langer, 1923) and its corollary. And we shall clearly distinguish between the different forms of energy contributing to E.

\section{2 | THE COSMIC SPACETIME}

Theoretical cosmology is founded on the Cosmological Principle (CP), comprising two essential symmetries: homogeneity and isotropy, at least on spatial scales (Yadav, Bagla, \& Khandai 2010) larger than $\sim 300 \mathrm{Mpc}$. An important consequence of this principle is the highly simplified form of the (FLRW) metric used to describe the cosmic spacetime (Melia, 2020), normally expressed as

$$
d s^{2}=c^{2} d t^{2}-a^{2}(t)\left[d r^{2}\left(1-k r^{2}\right)^{-1}+r^{2}\left(d \theta^{2}+\sin ^{2} \theta d \phi^{2}\right)\right] .
$$

This form is written in terms of comoving coordinates, in which $t$ is the proper (sometimes called 'cosmic') time, $r$ is the radius, $\theta$ is the poloidal angle and $\phi$ is the azimuth. Unless an object in the Universe exhibits so-called 'peculiar' motion, its comoving coordinates remain fixed. Proper motion and the universal expansion itself are instead given in terms of the proper radius, $R=a(t) r$, in which the expansion factor $a(t)$ is solely a function of time, not position. The most important element in this equation for this paper is the spatial curvature constant $k$, whose value is +1 for a closed universe, 0 for a flat, open universe, and -1 for an open universe. In reality, $k$ could be any real number, though one typically renormalizes the radius $r$ in any given cosmological model in order to reduce it to one of these three distinct integer values.

If one accepts the FLRW metric as the basis of our cosmology, most of the work in building a framework for the origin and evolution of the Universe revolves around the behavior of $a(t)$. Each of the models we have today assumes some combination of matter and energy, predicting its own distinct time dependence of the expansion factor. And in the majority of cases, the underlying dynamical equations for $a(t)$ are obtained by introducing the metric in Equation (11) into Einstein's field equations of general relativity (Melia. 2020). The one most relevant to us here is the Friedmann equation,

$$
H^{2} \equiv\left(\frac{\dot{a}}{a}\right)^{2}=\frac{8 \pi G}{3 c^{2}} \rho-\frac{k c^{2}}{a^{2}},
$$

in which an overdot denotes a derivative with respect to $t, \rho$ represents the total energy density in the cosmos, and $H \equiv \dot{a} / a$ is the Hubble parameter. In preparation for our discussion in $\S \mathrm{IV}$, we emphasize here that the Friedmann equation is exact. No approximations were made in deriving it directly from the full expression of Einstein's equations in general relativity.

\section{3 | THE FLATNESS PROBLEM}

Over the past several decades, precision measurements (Planck Collaboration et al. 2016, Spergel et al. 2003) of the cosmic microwave background (CMB) have shown that the Universe is very nearly flat, perhaps completely flat, with an inferred spatial curvature constant $k \approx 0$. Therefore, the density $\rho$ appears to be at (or very near) the so-called 'critical' density

$$
\rho_{c} \equiv \frac{3 c^{2} H^{2}}{8 \pi G}
$$

obtained by setting $k=0$ in Equation (2).

A density close to $\rho_{c}$ can be difficult to explain, however, unless $k$ has in fact always been exactly zero. To see why, let us define

$$
\Omega \equiv \rho / \rho_{c},
$$

and re-write Equation (2) as follows:

$$
1=\Omega(t)-\frac{k c^{2}}{\dot{a}^{2}} .
$$


Using subscript ' 0 ' to denote quantities pertaining to the present time $t_{0}$, we therefore find that

$$
\Omega(t)-1=\left(\frac{\dot{a}_{0}}{\dot{a}}\right)^{2}\left(\Omega_{0}-1\right) .
$$

This equation may not look very peculiar, but let's focus on the multiplicative factor $\left(\dot{a}_{0} / \dot{a}\right)^{2}$ on the right-hand side. Today, we've all become accustomed to the idea that the Universe may be accelerating, but this was not the situation at early times; it was actually decelerating at an enormous rate. Let's say for simplicity that $\rho$ was dominated by radiation. This would certainly have been the case during the first few hundred thousand years, though not afterwards. One may then put $\rho \propto a^{-4}$ in Equation (2), since the energy density of a radiation field scales inversely with the spatial volume ( $\left.\propto a^{3}\right)$, and inversely with the wavelength $(\alpha a)$, due to redshifting as the Universe expands. One can therefore easily see from the solution to Equation (2) that $a(t) \propto t^{1 / 2}$, as long as $k$ is small. (Incidentally, had we assumed $\rho$ was dominated by matter instead, we would have found that $a(t) \propto t^{2 / 3}$, but such differences have no impact on this argument.) The prefactor in Equation (6) would thus be $\left(\dot{a}_{0} / \dot{a}\right)^{2} \sim t / t_{0}$. So comparing the quantity $\Omega(t)-1$ at the GUT (Grand Unification Theory) time $\left(t_{\text {GUT }} \sim 10^{-35}\right.$ seconds; see below) with its counterpart 13.5 billion years later, we see that it must have been $\sim 10^{48}$ times smaller compared to its value today. Any indication that $\Omega_{0} \approx 1$ today therefore suggests that $\Omega(t)$ must have been fine tuned to nearly exactly 1 as $t \rightarrow 0$.

This fine-tuning is the origin of the so-called 'flatness' problem in cosmology, in the sense that it seems to require yet one more special initial condition without any physical explanation (Hawking, 1974; Lightman, 1990). But this clearly stems from the view that the Universe could have been born with any value of $k$, so an initial condition $\Omega(t)=1$ with $k \neq 0$ would appear to be highly unlikely. To address this issue, several different approaches have been taken to explain why the Universe appears to be flat (or nearly flat) today, in spite of it possibly having begun with an arbitrary spatial curvature.

One possible solution is to again invoke the anthropic principle (Collins \& Hawking, 1973), arguing that only a Universe with the correct density to form galaxies and stars would give rise to intelligent observers who would then inquire about the initial conditions. It should then not be surprising that we do not live in other types of universes with unsuitable values of $\Omega(t)$ at the beginning.

But the explanation that currently enjoys the most widespread acceptance (if not outright belief) is inflation, a period of exponential growth in the Universe only $\sim 10^{-35}$ seconds after the Big Bang, possibly associated with the separation of the strong and electroweak forces at the aforementioned GUT scale. In his paper, Guth (1981) proposed that such an early phase of very rapid expansion would solve several puzzles in cosmology, including the flatness problem. A requirement of inflation is that the density $\rho$ remain constant for a brief, though critical, period, and it is not difficult to see from Equation (2) that, under such circumstances, $a(t)$ would grow exponentially (again ignoring any small value of $k$ ). Thus, any value of $\Omega(t)-1$ at the beginning would rapidly shrink to near zero by the end of inflation, due to the enormously large factor $\dot{a} \sim e^{H t}$ in the r.h.s. denominator of Equation (6). And the Universe would emerge back into the standard hot Big Bang configuration to resume its expansion resulting in $\Omega_{0} \sim 1$ today. In other words, inflation could in principle wash out any initial condition associated with $\Omega(t)$ resulting from an arbitrary value of $k$.

Inflationary theory is not universally accepted, however, and recent observations are starting to raise doubt about whether it can actually explain the various horizon problems plaguing the standard model (Melia, 2018), and the fluctuation spectrum seen in the CMB (Liu \& Melia 2020). There are still too many gaps in the theory, and the continued refinement of cosmological measurements may eventually disprove it. For example, even after four decades of development, inflationary cosmology still lacks specificity regarding the field that is driving it (Bird, Peiris, \& Easther 2008). The various versions of the theory spawned by this ambiguity contain parameters and initial conditions that themselves require fine-tuning (Ijjas, Steinhardt, \& Loeb 2014), analogously to how 'unnatural' the initial density would be in the absence of inflation (see Eq. 6).

There are therefore good reasons to explore other explanations for the flatness of the Universe, even more so today because the ever improving observations are telling us that $k$ is not only close to zero, but is probably exactly zero. So the "flatness' problem may be moot. It may not be a problem at all, if it turns out that there is a physical reason why the Universe had to have perfect spatial flatness as one of its initial conditions.

\section{4 | COSMIC ENERGY}

Today, we have a better understanding of what $k$ stands for. Spatial curvature is not merely a geometric property of the spacetime - it results from a well defined physical attribute, which can be understood with a more thorough inspection of the Friedmann Equation (2). Though this expression derives from Einstein's field equations, it is not difficult to find a simple heuristic argument for its physical meaning, which includes an explanation for the spatial curvature constant $k$. This is most easily done by invoking the corollary to the BirkhoffJebsen theorem (Birkhoff \& Langer. 1923), which allows us to understand why the influence of any isotropic, external source of gravity completely cancels within a spherical cavity. This theorem is a relativistic generalization of Newton's theorythat the gravitational field outside a spherically symmetric 
body is indistinguishable from that of the same mass concentrated at its center. It was first pointed out by Weinberg (1972) that, due to this symmetry, a limited use of Newtonian gravity is permitted for some cosmological applications, such as the one we are concerned with here.

A consequence of isotropy is that every observer in the cosmos experiences net zero acceleration due to the surrounding mass. But in fact the relative acceleration between any two points is not zero; it depends on the mass/energy content between them. This is the reason why the corollary to the Birkhoff-Jebsen theorem is so germane to our discussion, because it describes the field inside an empty spherical cavity at the center of an isotropic distribution, which may be used to calculate the relative acceleration locally. The metric inside such a cavity is Minkowskian, a situation not unlike we find in electromagnetism, where we would calculate the electric field inside a spherical cavity embedded within an otherwise uniform charge distribution to be zero. So even in the classical limit, it is safe to argue that the medium exterior to a sphere can be represented as a sequence of shells with an ever increasing radius, each of which produces zero effect within the cavity.

Let us imagine 'carving' a sphere of radius $R_{\mathrm{s}}(t)=a(t) r_{\mathrm{s}}$ out of an otherwise homogeneous, isotropic universal medium (fig. 1. The rest of the Universe has zero gravitational influence inside this cavity. We then fill the sphere with density $\rho$ all the way up to an infinitesimal distance away from $R_{\mathrm{s}}(t)$. According to the Cosmological Principle, this density needs to be a function solely of $t$. In addition, the FLRW metric tells us that every pair of points within and outside the sphere recede from each other at a rate proportional to the expansion factor $a(t)$, which itself is the same everywhere. But according to the Birkhoff-Jebsen theorem and its corollary, we only need to consider the source of gravity within $R_{s}$ to determine the local dynamics of this region (i.e., the behavior of $a$ ) extending out to this radius.

We place an observer at the center of this sphere, where they calculate the kinetic energy of a shell with thickness $d R$ at $R$ to be

$$
d K=4 \pi R^{2} d R \frac{\rho(t) \dot{R}^{2}}{2 c^{2}},
$$

where the factor $c^{2}$ converts the energy density to a mass density. One must be aware, of course, that there are limitations to how far one can take this classical, heuristic approach. Inferring a mass density using this method for the purpose of calculating a "kinetic" energy is reasonable for a matter dominated Universe, perhaps even a matter plus radiation Universe, as long as the radiation in the latter is 'trapped' within the matter, allowing one to use the $E=m c^{2}$ conversion to infer the gravitational mass. This approach would not be sensible for a purely radiation-dominated Universe, however. If the observer now integrates this quantity from $r=0$ (their location) to $r=r_{\mathrm{s}}$ (the edge of the sphere), they find that the total kinetic

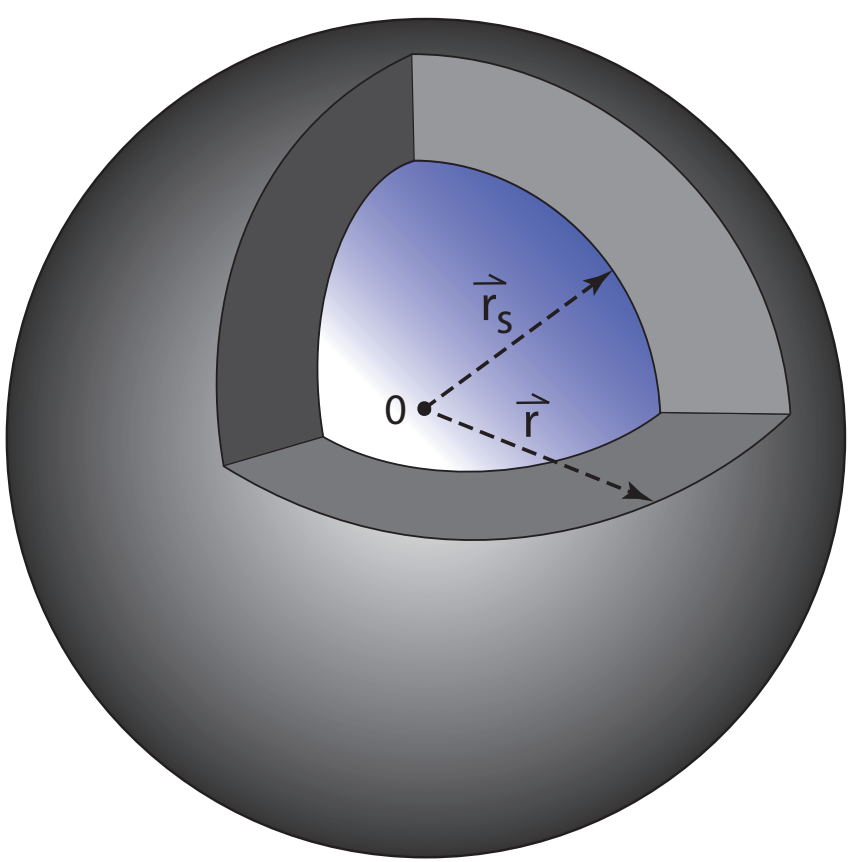

FIGURE 1 The Birkhoff-Jebsen theorem Birkhoff \& Langer 1923) and its corollary tell us that in an isotropic Universe, the mass-energy everywhere outside (gray) of a spherical region (blue) of radius $r_{s}$ (i.e., at all $r>r_{s}$ ) has no gravitational influence at $r \leq r_{s}$. Thus, the gravitational acceleration of any point on the shell at $r_{s}$ relative to an observer at the origin depends solely on the mass-energy contained within this (blue) sphere.

energy of the sphere relative to them is

$$
K=\frac{2 \pi}{5} \frac{\rho(t)}{c^{2}} a^{3} \dot{a}^{2} r_{\mathrm{s}}^{5} .
$$

This sphere also has gravitational energy (remember this is a classical derivation). The observer finds that the corresponding potential energy of a shell at $R$ is

$$
d U=-4 \pi R^{2} d R \frac{G M(R) \rho(t)}{c^{2} R},
$$

where

$$
M(R)=\frac{4 \pi}{3} R^{3} \frac{\rho(t)}{c^{2}}
$$

is the total mass enclosed within this radius. Thus, after integrating this quantity from $r=0$ to $r_{\mathrm{s}}$, they find that the total potential energy of the sphere (relative to them at the origin) is

$$
U=-\frac{16 \pi^{2} G}{15} \frac{\rho(t)^{2}}{c^{4}} a^{5} r_{\mathrm{s}}{ }^{5} .
$$

Since they are doing this calculation classically, they therefore conclude that the sphere expanding away from them has a 
net kinetic plus gravitational energy

$$
E=\frac{2 \pi}{5} \frac{\rho(t)}{c^{2}} a^{3} \dot{a}^{2} r_{\mathrm{s}}^{5}-\frac{16 \pi^{2} G}{15} \frac{\rho(t)^{2}}{c^{4}} a^{5} r_{\mathrm{s}}^{5},
$$

and if they re-arrange this slightly, a very recognizable form appears:

$$
\left(\frac{\dot{a}}{a}\right)^{2}=\frac{8 \pi G}{3 c^{2}} \rho(t)+\frac{5 c^{2} E}{2 \pi \rho(t) a^{5} r_{\mathrm{s}}^{5}} .
$$

The local conservation of energy relative to the observer at the origin is thus simply the Friedmann Equation (2), so long as one interprets the spatial curvature constant to be

$$
k \equiv-\frac{10}{3 r_{\mathrm{s}}^{2}}\left(\frac{\epsilon}{\rho}\right),
$$

written in terms of the local kinetic plus gravitational energy density

$$
\epsilon \equiv \frac{3 E}{4 \pi R_{\mathrm{s}}^{3}},
$$

and the energy density $\rho$ in the cosmic fluid. But note that $\epsilon$ does not include $\rho$ itself. Incidentally, calling the quantity $k$ defined in Equation (14) a constant would be justified only as long as $\epsilon$ and $\rho$ scale in proportion to each other. In a classical context, this simply means that the sum of kinetic plus gravitational energy (i.e., $E$ ) is conserved, which is what one would expect without an energy non-conserving influence affecting the cosmic expansion. One must be careful in general relativity, however, since the energy measured by an observer can change in a time-dependent spacetime. As we shall discuss shortly, however, even this distinction may not matter for our interpretation of $k$ if $\epsilon$ is strictly zero, because then $\epsilon$ would always be zero in both the classical and relativistic contexts.

We thus see that a Universe with positive spatial curvature $(k>0)$ contains negative net energy $(\epsilon<0)$ and is therefore bounded-for which the more common terminology is that the Universe is 'closed'. Negative spatial curvature arises when this energy is positive $(\epsilon>0)$, which characterizes an unbounded (or 'open') Universe. A Universe with zero net energy $(\epsilon=0)$ is spatially flat $(k=0)$, but also 'open'. To put this in context, let us consider the following simple analogy. Imagine throwing a ball vertically into the air, ignoring any resistance. Its motion will depend on how its initial speed $\left(v_{\text {init }}\right)$ compares to the value it needs $\left(v_{\text {esc }} \equiv \sqrt{2 G M_{\text {Earth }} / R_{\text {Earth }}}\right)$ to escape from Earth. If $v_{\text {init }}<v_{\text {esc }}$, the ball slows down, stops and then falls back to its point of origin. The ball is bounded to Earth because its total energy is negative. If $v_{\text {init }}>v_{\text {esc }}$, the ball has positive energy and is unbounded. It will continue receding to infinity with a speed always greater than zero. The critical initial condition is $v_{\text {init }}=v_{\text {esc }}$, for which the ball rises indefinitely, though with an ever decreasing speed, coming to rest at infinity.

A spatially flat Universe therefore has zero local energy density $\epsilon$. Normally, attempts at quantitatively handling gravitational energy in general relativity face the daunting problem of localization (Landau 1962). Here, however, the $\epsilon=0$ condition holds at every point in the Universe, so it follows that the total kinetic plus gravitational energy must also be zero, no matter how one chooses to do the integration. This simplified heuristic argument therefore teaches us that spatial flatness is a unique, fundamental physical property of the Universe, which apparently contains equal portions of positive kinetic (expansion) energy and negative gravitational energy. Moreover, $k$ is constant, so this perfect balance must have been present from the beginning, constituting an important initial condition at the time of the Big Bang.

\section{5 | DISCUSSION}

It is tempting to think that perhaps our Newtonian approach may lack certain nuances manifested solely via the full general relativistic treatment. But remember that our calculation of $\epsilon$ has been carried out strictly on a local basis, with full justification from the Birkhoff-Jensen theorem. In fact, the proper radius of the sphere used in this construction, i.e., $a(t) r_{s}$, may be chosen arbitrarily small compared to the Hubble radius $R_{\mathrm{h}}=c / H$, because we are interested merely in the local value of $\epsilon$, point by point. And once $\epsilon$ is known for a given observer, every spacetime point in the FLRW cosmos has precisely the same value, at least according to the Cosmological principle. There is therefore nothing missing from Equation (13) that would otherwise emerge from an analogous calculation using Einstein's equations. In particular, notice that this equation matches precisely the actual Friedmann equation (2) derived from general relativity.

The key result of this derivation is a clear, unambiguous identification of the energy $E$ in Equation (13), which appears to be directly proportional to the spatial curvature constant $k$. It includes only kinetic plus gravitational energy, but not a contribution from $\rho$ itself. Our result therefore agrees completely with that of Faraoni and Cooperstock (2003) in the case of $k=0$, who used an entirely different method of identifying the kinetic and potential energy of the cosmic expansion. In both cases, the conclusion is that $E$ is zero, but it only represents the kinetic and gravitational energy. Thus, if the latest observations are telling us that $k=0$, we cannot avoid the conclusion that the Universe has net positive energy, when the integrated value of $\rho$ is taken into account.

Nevertheless, one must still contend with several possible caveats to this result. First, it may turn out that $k$ is close to, but not exactly, zero, in which case there may still be some wiggle room to keep the overall sum of energies zero. We note, in this regard, that the most precise measurement of the spatial curvature to date, from the Planck mission (Planck Collaboration et al. 2020), is quoted as $\Omega_{k}=0.001 \pm 0.002$, where 
$\Omega_{k}$ is defined as the last term in Equation (2) divided by $H^{2}$. There is no question this parameter is fully consistent with zero, but it may still allow for a very slight positive or negative bias. This condition is the essential caveat with this work, for if it turns out that $k$ is in fact not exactly zero, then the conclusions we draw here become moot. We note, in this regard, that observations other than those pertaining to the $\mathrm{CMB}$ also indicate a likely flat Universe, consistent with Planck (Wei \& Melia 2020a 2020b), though the precision of these alternative measurements is inferior to that of the CMB satellite missions. Nevertheless, one must keep in mind that any discussion relating the total energy of the Universe to its spatial flatness becomes much more difficult to resolve if $k$ is not zero, given in part by the complications discussed earlier of handling the energy in a general relativistic framework when the spacetime curvature changes with time.

Second, inflation may have happened after all, in which case $\Omega_{0}-1$ in Equation (6) is close to zero today only because of the significant dilution that took place during the inflationary expansion at $t \sim 10^{-35} \mathrm{sec}$. Having said this, it is also possible that $k=0$ was an initial condition regardless of whether inflation happened or not, in which case our inference on the total energy would be valid nonetheless. It is beyond the scope of the present paper to discuss inflation at greater length, but it might be helpful to point out that the observational evidence in favor of such an event having occurred in the early Universe is rather slim. As noted earlier, the latest analysis of the Planck data suggests that a slow-roll inflaton potential could not simultaneously have solved both the CMB temperature horizon problem and provided a mechanism for seeding the primordial fluctuation spectrum Liu and Melia (2020). And at a more fundamental level, it is even debatable whether these quantum fluctuations could have classicalized to produce the classical large-scale structure we see today Melia (2021).

Third, in addition to inflation, there are other possible reasons why the Universe may appear close to spatial flatness today, even if $k=0$ was not an initial condition. For example, a key factor in many 'cyclic' models of the Universe is a so-called ekpyrotic phase, in which the contraction preceding the Big Bang evolves ultra-slowly (Ijjas \& Steinhardt, 2019; Raveendran \& Sriramkumar. 2019). In such models, it is this ekpyrotic phase that can explain the smoothness and flatness of the Universe on large scales, and it can also generate a distinctive primordial spectrum of scalar and tensor fluctuations.

So there are still many unknowns, and while our conclusions in this paper are suggestive, we would not call them 'final'. We are beginning to get some guidance from the data concerning the nature of the Big Bang, but there is still much work to do before we can identify its true physical origin.

\section{6 | CONCLUSION}

In a way, this result completes the derivation begun by Tryon in his seminal paper (Tryon 1973), in which he attempted to balance the gravitational energy by the rest mass energy in the cosmic fluid. As we now understand it, the kinetic energy of expansion exactly balances its gravitational counterpart, leaving $\rho$ as an 'excess' positive contribution to $E$.

If these results withstand the test of time, it would not be possible to reconcile the presently 'measured' non-zero energy of the Universe with the requirements of a quantum birth from 'nothing'. Perhaps a quantum fluctuation in a preexisting vacuum might still be consistent, but one would need to understand how its energy $E$-in the context of our Universe's measured value of the Planck constant, $\hbar$ - could have allowed it to grow and classicalize and live long enough for us to see it today. Would this be an indication that $\hbar$ had to be different prior to the Big Bang?

\section{Author contributions}

Fulvio Melia is the sole author of this paper.

\section{Financial disclosure}

None reported.

\section{Conflict of interest}

The authors declare no potential conflict of interests.

\section{REFERENCES}

Albrow, M. G. (1973, January), Nature Physical Science, 241(107), 56-57. doi:

Barrow, J. D., \& Tipler, F. J. 1988, The anthropic cosmological principle.

Bird, S., Peiris, H. V., \& Easther, R. (2008, October), Phys. Rev. D, 78(8), 083518. doi:

Birkhoff, G. D., \& Langer, R. E. 1923, Relativity and modern physics.

Collins, C. B., \& Hawking, S. W. (1973, March), ApJ, 180, 317-334. doi:

Cooperstock, F. I. (1994, March), General Relativity and Gravitation, 26(3), 323-327. doi:

Ellis, G. F. R. (2006, February), arXiv e-prints, astro-ph/0602280.

Faraoni, V., \& Cooperstock, F. I. (2003, April), ApJ, 587(2), 483-486. doi:

Guth, A. H. (1981, January), Phys. Rev. D, 23(2), 347-356. doi:

Hawking, S. W. (1974, January), The anisotropy of the universe at large times. In M. S. Longair (Ed.), Confrontation of Cosmological Theories with Observational Data Vol. 63, p. 283-286.

He, D., Gao, D., \& Cai, Q.-y. (2014, April), Phys. Rev. D, 89(8), 083510. doi: 
Ijjas, A., \& Steinhardt, P. J. (2019, August), Physics Letters B, 795, 666-672. doi:

Ijjas, A., Steinhardt, P. J., \& Loeb, A. (2014, September), Physics Letters B, 736, 142-146. doi:

Isham, C. (1994), Cosmos and Creation. In Quantum Cosmology and the Origin of the Universe, Lecture presented. Cambridge University.

Khoury, J., Ovrut, B. A., Steinhardt, P. J., \& Turok, N. (2001, December), Phys. Rev. D, 64(12), 123522. doi:

Kohli, I. S. (2016), arXiv e-prints, arXiv:1405.6091.

Krauss, L. M. 2012, A Universe from Nothing: Why There is Something Rather than Nothing.

Landau, E. M., L. D. \& Lifshitz. 1962, The Classical Theory of Fields.

Lightman, R., A. \& Brawer. (1990), The first published reference to a 'flatness' problem in cosmology appears to have been in Robert Dicke's Jayne Lectures of 1969. The origin of the problem was discussed by Dicke during an interview for a subsequent book by A. Lightman \& R. Brawer. In Origins: The Lives and Worlds of Modern Cosmologists. Harvard University Press.

Linde, A. (2007), Inflationary Cosmology. In M. Lemoine, J. Martin, \& P. Peter (Eds.), Lecture Notes in Physics, Berlin Springer Verlag Vol. 738, p. 1. doi:

Liu, J., \& Melia, F. (2020, July), Proceedings of the Royal Society of London Series A, 476(2239), 20200364. doi:

Melia, F. (2012, May), MNRAS, 422(2), 1418-1424. doi:

Melia, F. (2018, September), European Physical Journal C, 78(9), 739. doi:

Melia, F. 2020, The Cosmic Spacetime.

Melia, F. (2021, July), Physics Letters B, 818, 136362. doi:

Planck Collaboration, Ade, P. A. R., Aghanim, N. et al. (2016, September), $A \& A, 594$, A13. doi:

Planck Collaboration, Aghanim, N., Akrami, Y. et al. (2020, September), $A \& A, 641$, A6. doi:

Raveendran, R. N., \& Sriramkumar, L. (2019, February), Phys. Rev. D, 99(4), 043527. doi:

Rosen, N. (1994, March), General Relativity and Gravitation, 26(3), 319-321. doi:

Spergel, D. N., Verde, L., Peiris, H. V. et al. (2003, September), ApJS, 148(1), 175-194. doi:

Tolman, R. C. (1931, November), Physical Review, 38(9), 17581771. doi:

Tryon, E. P. (1973, December), Nature, 246(5433), 396-397. doi:

Vilenkin, A. (1982, November), Physics Letters B, 117(1-2), 25-28. doi:

Šekrst, K. (2020), Much Ado About Nothing: Cosmological and Anthropic Limits of Quantum Fluctuations. In L. Borši, D. Poljak, I. S. Karasman, et al. (Eds.), Physics and Philosophy II pp. 105114. Institute for Philosophy Zagreb.

Wei, J.-J., \& Melia, F. (2020a, July), ApJ, 897(2), 127. doi:

Wei, J.-J., \& Melia, F. (2020b, January), ApJ, 888(2), 99. doi:

Weinberg, S. 1972, Gravitation and Cosmology: Principles and Applications of the General Theory of Relativity.

Yadav, J. K., Bagla, J. S., \& Khandai, N. (2010, July), MNRAS, 405(3), 2009-2015. doi:

Zeldovich, Y. B., \& Starobinskij, A. A. (1984, June), Pisma $v$ Astronomicheskii Zhurnal, 10, 323-328.

\section{AUTHOR BIOGRAPHY}

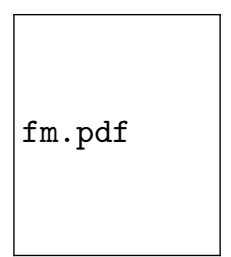

Fulvio Melia is an internationally recognized leader in astrophysics and cosmology, having previously published seven books in physics and astronomy, and approximately 400 refereed publications in the these fields. He has also served as associate editor of the Astrophysical Journal and Astrophysical Journal Letters. 